\title{
Wavelet Analysis of Longitudinal Impact Responses
}

\author{
Peter Eberhard \\ Institute of Applied Mechanics, University of Erlangen, Egerlandstr. 5, D-91058 Erlangen, Germany
}

\begin{abstract}
Bin $\mathrm{Hu}$ and Zhuo Li
Institute B of Mechanics, University of Stuttgart, Pfaffenwaldring 9, D-70550 Stuttgart, Germany
\end{abstract}

(Received 9 November 2000; accepted 14 November 2001)

\begin{abstract}
The dynamics of a mechanical system during impact and after impact has very different characteristics since impact responses are transient and have very high frequency components. For the numerical simulation of impact responses, the discretisation of elastic bodies plays an important role in modal analysis and in the finite element method. In this paper, we use wavelet analysis as a tool to investigate the influence of the discretisation on impact responses. As an example, a longitudinal impact system of a rigid body striking an elastic rod is used. We discuss how well the impact response of this continuous impact system is approximated using a discrete system with finite degrees of freedom based on the modal approach from a wavelet analysis point of view. Numerical simulation results are compared with experimental results. Furthermore, in mechanical systems weak collisions may appear. They are difficult to detect experimentally since motion measurements are often available only partially and measured signals are always disturbed by measurement noise. It is shown that wavelet analysis is a powerful tool to detect weak collisions even from noisy signals.
\end{abstract}

\section{INTRODUCTION}

The behaviour of a mechanical system under impact loading is a subject of great interest in engineering mechanics. Impact responses are nonstationary signals which contain very high frequency components during the short duration of impact and lower frequency components in their long time behaviour after impact. For analysis of impact responses, wavelets provide a useful tool. They allow the changes in the spectral composition of impact responses to be obtained and presented in the form of a time-scale map. ${ }^{1,2}$ In numerical simulations of impact responses the discretisation of elastic bodies plays an important role in the modal approach and in the finite element method. In this paper, we use wavelet analysis as a tool to investigate the influence of the discretisation on impact responses. As an example, a longitudinal impact system of a ball striking a free rod is used. We discuss how well the impact response of this continuous impact system is approximated using a discrete system with finite degrees of freedom based on the modal approach from a wavelet analysis point of view. While traditional comparisons in the time domain give no frequency localisation and traditional comparisons in the frequency domain give no time localisation, comparisons in the time-scale map by means of the wavelet analysis provide both time and frequency localisation simultaneously. Compared with the short time Fourier transformation, the wavelet analysis offers a good time resolution at high frequencies and a good frequency resolution at low frequencies.

Moreover, the wavelet analysis can also be used for denoising. In mechanical systems weak collisions may appear. For the failure analysis, the time localisation of the collisions provides important information. However, weak collisions are difficult to detect since motion measurements are not al- ways completely available and measured signals are always disturbed by measurement noise. In this paper, the collision detection from noisy signals using the wavelet analysis is also discussed for longitudinal impacts of a rigid body striking a fixed rod.

\section{LONGITUDINAL IMPACT DYNAMICS}

Longitudinal impact responses, e.g. created by a ball striking an elastic rod, are typical nonstationary signals. During the short duration of impact, impact responses have very high frequency components, which decay quickly after separation and low frequency components are then dominant. Wavelets may provide a powerful tool for the analysis of such nonstationary impact responses. The schematic description of the impact system and the experiment is shown in Fig. 1. A slender homogeneous brass rod is struck at the contact point $x=0$ by a moving steel ball with mass $m_{1}=0.11 \mathrm{~kg}$ and velocity $v_{0}=0.886 \mathrm{~m} / \mathrm{s}$. The axial displacement and velocity of the rod at position $x$ and time $t$ are denoted by $u(x, t)$ and $v(x, t)$, respectively. The brass rod has Young's modulus $E=10^{5} \mathrm{~N} / \mathrm{mm}^{2}$, Poisson's ratio $\nu=0.36$, density $\rho=8430 \mathrm{~kg} / \mathrm{m}^{3}$, cross-sectional area $A=\pi \mathrm{cm}^{2}$, length $L=1.488 \mathrm{~m}$ and mass $m=\rho A L=3.941 \mathrm{~kg}$. The velocity of the longitudinal wave propagation along the brass $\operatorname{rod}$ is $c=\sqrt{E / \rho}=3444.2 \mathrm{~m} / \mathrm{s}$. The rod may move freely in the horizontal direction and is initially at rest. It is assumed that transverse motions of the rod and the vibration of the ball can be neglected.

For our measurements, advanced instruments, nowadays available for measuring high frequency impact responses, were used. For the measurement of displacements and velocities, a Laser-Doppler-Vibrometer of type Polytec OFV-3000/ OFV-302 was used which utilises an interferometric techniques to measure vibrational signals and is capable of detecting such signals of subnanometer amplitude. In the meas- 